\title{
A Hybrid Structure Solution of Quaternion Lyapunov Equation and Its Optimal Approximation
}

\author{
Wang Yun, Huang Jingpin ", Lan Jiaxin \\ College of Science, Guangxi University for Nationalities, Nanning, China \\ Email address: \\ wang_yun_1125@163.com (Wang Yun), hjp2990@126.com (Huang Jingpin),674075139@qq.com (Lan Jiaxin) \\ ${ }^{*}$ Corresponding author
}

To cite this article:

Wang Yun, Huang Jingpin, Lan Jiaxin. A Hybrid Structure Solution of Quaternion Lyapunov Equation and Its Optimal Approximation. American Journal of Applied Mathematics. Vol. 7, No. 1, 2019, pp. 30-36. doi: 10.11648/j.ajam.20190701.15

Received: April 22, 2019; Accepted: May 24, 2019; Published: June 15, 2019

\begin{abstract}
Recently, the establishment of a multi-structure control system has demonstrated vital significance in practice. Its stability analysis are mostly determined by Lyapunov matrix equation. Tridiagonal-arrow matrix (TA matrix for short) is a special matrix with hybrid structure. In this paper, the problem of TA constraint solution to continuous Lyapunov equation $A^{*} X+X A=C$ over quaternion field is discussed. By using the representation of vectors of a TA matrix and Kronecker product of matrices, a constrained problem will be transformed into an unconstrained equation. Then the necessary and sufficient conditions for the equation with TA and self-conjugate TA solutions as well as the expression of general solution are obtained. Meanwhile, when the solution set is nonempty, by using invariance of Frobenius norm of orthogonal matrix product, the optimal approximation solution with minimal Frobenius norm for a given TA matrix is derived. Finally, two numerical examples are provided to verify the algorithm.
\end{abstract}

Keywords: Quaternion Field, Lyapunov Equation, Tridiagonal-Arrow Matrix, Kronecker Product, Optimal Approximation

\section{Introduction}

The stability of many control systems in modern control theory is ultimately analyzed through the solution to Lyapunov matrix equation, thus enjoying an extensive application [1-3]. According to different problems in practice, Lyapunov equation can be classified into mixed, continuous and discrete equations, which have generated fruitful results in the complex field and quaternion field [4-9]. For example, by using the complex representation operator of the quaternion matrices, the sub-positive definite solutions and iterative algorithm with parameter of mixed quaternion Lyapunov equation were discussed [8]. The sufficient and necessary conditions of a circulant solution for the unified algebraic Lyapunov equation and its general solution expressions were given in [9]. However, research concerning multi-structure constrained solution of these matrix equations over quaternion field is scarce.

As a special matrix, tridiagonal matrix is widely used in medicine, signal processing, engineering and other fields. Its important properties such as eigenvalue, eigenvector, inverse matrix, determinant, singular value decomposition and stability are hot topics, which mainly results from its important status in numerical calculation. For example, in eigenvalues calculate, a symmetric matrix can be transformed into a symmetrical tridiagonal matrix by Lanczos algorithm [10-11]. Tridiagonal matrix is also involved in building cubic spline interpolation function in ship mathematical lofting, and solving boundary value problems of differential equations [12-13].

Arrow matrix is also a special matrix of vital significance in control theory. For instance, in the non-linear regulating system, the parametric matrix of the governing equations is usually an arrow matrix. Recently, there are some related researches on the eigenvalues and constraint equations of arrow matrix. Ma and Huang proposed the method of constructing quaternion arrow matrix from two right eigenpairs [14]. Huang et al. [15] discussed the solutions of the quaternion Sylvester equation with arrow matrix constraints. The authors investigated the hybrid structure of tridiagonal matrix and arrow matrix in real field as well as its inverse eigenvalue problem [16].

In this paper, we consider the quaternion continuous 
Lyapunov equation

$$
A^{*} X+X A=C,
$$

where $A, C \in \mathrm{Q}^{n \times n}$ are given quaternion matrices, and $X \in \mathrm{Q}^{n \times n}$ is an unknown quaternion matrix.

The purpose of this article is to discuss the tridiagonalarrow matrix solution of equation (1) and its optimal approximation over quaternion field.

For convenience, let $\mathrm{R}^{n \times n}, \mathrm{C}^{n \times n}, \mathrm{Q}^{n \times n}$ be the set of all $n \times n$ real matrices, the set of all $n \times n$ complex matrices, the set of all $n \times n$ quaternion matrices, respectively. For $A^{T}, \bar{A}, A^{*}, A^{+}$denote the transpose matrix, the conjugate matrix, the conjugate transpose matrix and the Moore-Penrose generalized inverse of a matrix $A$, respectively. $\operatorname{vec}(A)$ denotes that $A$ straightens vectors in column order. $A \otimes B$ stands for the Kronecker product of $A$ and $B .\|A\|=\sqrt{\operatorname{tr}\left(A^{*} A\right)}$ stands for the Frobenius norm.

Definition 1 . Let $1 \leq s<n$, the matrix given by

$$
T_{n}(s)=\left[\begin{array}{ccccccc}
a_{1} & c_{1} & & & & & \\
b_{1} & \ddots & \ddots & & & & \\
& \ddots & a_{s-1} & c_{s-1} & & & \\
& & b_{s-1} & a_{s} & c_{s} & \cdots & c_{n-1} \\
& & & b_{s} & a_{s+1} & & \\
& & & \vdots & & \ddots & \\
& & & b_{n-1} & & & a_{n}
\end{array}\right] \in \mathrm{Q}^{n \times n},
$$

will be called a tridiagonal-arrow matrix (TA matrix for short). The set of all $n \times n$ quaternion TA matrices are represented by $\mathrm{T}_{n}(\mathrm{Q})$. In this case, if $b_{i}=\bar{c}_{i}, a_{i}=\bar{a}_{i}$, $i=1, \cdots, n-1$, then $\mathrm{T}_{n}(s)$ is called a quaternion self-conjugate TA matrix. The set of all $n \times n$ quaternion self-conjugate TA matrices are represented by $\mathrm{ST}_{n}(\mathrm{Q})$. Obviously, $\mathrm{T}_{n}(1)$ is an arrow matrix. $\mathrm{T}_{n}(n-1)$ is a tridiagonal matrix, $s$ is a controls parameter of $\mathrm{T}_{n}(s)$.

A TA matrix (2) is uniquely determined by $3 n-2$ tridiagonalarrow elements. Denote

$$
\begin{aligned}
& l(T)=\left(a_{1}, \cdots, a_{n}, b_{1}, \cdots, b_{n-1}, c_{1}, \cdots, c_{n-1}\right)^{T} \in \mathrm{Q}^{3 n-2},
\end{aligned}
$$

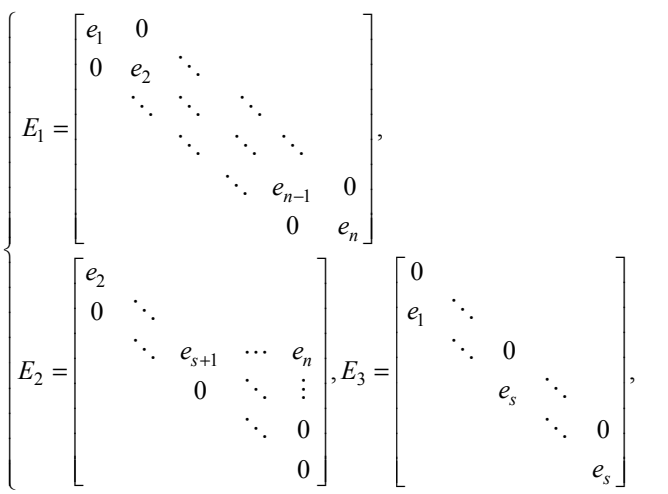

where $e_{i}$ is the $i$ th column of $I_{n}$, and let

$$
E=\left(E_{1}, E_{2}, E_{3}\right) \in \mathrm{R}^{n^{2} \times(3 n-2)} .
$$

Obviously, $E$ is column orthogonal, and it's easy to verify $E^{*} E=I_{3 n-2}$.

Lemma 1. Let $T=\left(a_{i j}\right)_{n \times n} \in \mathrm{Q}^{n \times n}$, then

$$
T \in \mathrm{T}_{n}(\mathrm{Q}) \Leftrightarrow \operatorname{vec}(T)=E \cdot l(T),
$$

where $l(T), E$ are represented as (3),(5).

Lemma 2 ([15]). The quaternion matrix equation $A X=B$ has a solution if and only if $A A^{+} B=B$. In this case, the general solution and the least square solution of equation can be expressed as $X=A^{+} B+\left(I-A^{+} A\right) Y$, where $Y \in \mathrm{Q}^{n \times n}$ is an arbitrary quaternion matrix, and have a unique minimal Frobenius norm least squares solution $\tilde{X}=A^{+} B$.

The problems are described in detail as follows.

Problem 1. Given $A, C \in \mathrm{Q}^{n \times n}$, find $X \in \mathrm{T}_{n}(Q)$ (or $\left.X \in \mathrm{ST}_{n}(Q)\right)$, such that $A^{*} X+X A=C$.

Problem 2. Let solution set $\Omega \neq \varnothing$ of problem 1, given $K \in \mathrm{T}_{n}(Q)$, find $\tilde{X} \in \Omega$, such that $\min _{X \in \Omega}\|X-K\|=\|\tilde{X}-K\|$.

\section{Solution of Problem 1}

First, we discuss the TA solution of equation (1). Let $\hat{X} \in \mathrm{T}_{n}(\mathrm{Q}), \hat{X}$ can be uniquely expressed as

$$
\hat{X}=X_{0}+X_{1} i+X_{2} j+X_{3} k
$$

where $X_{i} \in \mathrm{R}^{n \times n}(i=0,1,2,3)$ are real TA matrices. $A, C \in \mathrm{Q}^{n \times n}$ can be uniquely expressed as

$$
\left\{\begin{array}{l}
A=A_{0}+A_{1} i+A_{2} j+A_{3} k, \\
C=C_{0}+C_{1} i+C_{2} j+C_{3} k,
\end{array}\right.
$$

where $A_{i}, C_{i} \in \mathrm{R}^{n \times n}(i=0,1,2,3)$, so the quaternion Lyapunov equation (1) is equivalent to

$$
\begin{aligned}
& \left(A_{0}+A_{1} i+A_{2} j+A_{3} k\right)^{*}\left(X_{0}+X_{1} i+X_{2} j+X_{3} k\right) \\
& +\left(X_{0}+X_{1} i+X_{2} j+X_{3} k\right)\left(A_{0}+A_{1} i+A_{2} j+A_{3} k\right) \\
& =C_{0}+C_{1} i+C_{2} j+C_{3} k .
\end{aligned}
$$

By expanding the left side of the equation (9), and according to the uniqueness of the real decomposition of the quaternion matrix, we get

$$
\left\{\begin{array}{l}
A_{0}^{T} X_{0}+X_{0} A_{0}+A_{1}^{T} X_{1}-X_{1} A_{1}+A_{2}^{T} X_{2}-X_{2} A_{2}+A_{3}^{T} X_{3}-X_{3} A_{3}=C_{0}, \\
-A_{1}^{T} X_{0}+X_{0} A_{1}+A_{0}^{T} X_{1}+X_{1} A_{0}+A_{3}^{T} X_{2}+X_{2} A_{3}-A_{2}^{T} X_{3}-X_{3} A_{2}=C_{1}, \\
-A_{2}^{T} X_{0}+X_{0} A_{2}-A_{3}^{T} X_{1}-X_{1} A_{3}+A_{0}^{T} X_{2}+X_{2} A_{0}+A_{1}^{T} X_{3}+X_{3} A_{1}=C_{2}, \\
-A_{3}^{T} X_{0}+X_{0} A_{3}+A_{2}^{T} X_{1}+X_{1} A_{2}-A_{1}^{T} X_{2}-X_{2} A_{1}+A_{0}^{T} X_{3}+X_{3} A_{0}=C_{3},
\end{array}\right.
$$

where $X_{i} \in \mathrm{R}^{n \times n}(i=0,1,2,3)$ are real TA matrices, thus by 
lemma 1 , we have

$$
\operatorname{vec}\left(X_{i}\right)=E \cdot l\left(X_{i}\right) \quad(i=0,1,2,3),
$$

where $E$ is defined as (5). We write

$$
G=\left[G_{i j}\right]_{i, j=1}^{4}
$$

where $G_{i j}(i, j=1,2,3,4)$ as follows

$$
\begin{aligned}
& G_{11}=I \otimes A_{0}^{T}+A_{0}^{T} \otimes I, \quad G_{12}=I \otimes A_{1}^{T}-A_{1}^{T} \otimes I, \\
& G_{13}=I \otimes A_{2}^{T}-A_{2}^{T} \otimes I, \quad G_{14}=I \otimes A_{3}^{T}-A_{3}^{T} \otimes I, \\
& G_{21}=-I \otimes A_{1}^{T}+A_{1}^{T} \otimes I, \quad G_{22}=I \otimes A_{0}^{T}+A_{0}^{T} \otimes I, \\
& G_{23}=I \otimes A_{3}^{T}+A_{3}^{T} \otimes I, \quad G_{24}=-I \otimes A_{2}^{T}-A_{2}^{T} \otimes I, \\
& G_{31}=-I \otimes A_{2}^{T}+A_{2}^{T} \otimes I, \quad G_{32}=-I \otimes A_{3}^{T}-A_{3}^{T} \otimes I, \\
& G_{33}=I \otimes A_{0}^{T}+A_{0}^{T} \otimes I, \quad G_{34}=I \otimes A_{1}^{T}+A_{1}^{T} \otimes I, \\
& G_{41}=-I \otimes A_{3}^{T}+A_{3}^{T} \otimes I, \quad G_{42}=I \otimes A_{2}^{T}+A_{2}^{T} \otimes I, \\
& G_{43}=-I \otimes A_{1}^{T}-A_{1}^{T} \otimes I, \quad G_{44}=I \otimes A_{0}^{T}+A_{0}^{T} \otimes I, \\
& \tilde{G}=G\left[\begin{array}{llll}
E & & & \\
& E & & \\
& & E & \\
& & & E
\end{array}\right], L=\left[\begin{array}{c}
\operatorname{vec}\left(C_{0}\right) \\
\operatorname{vec}\left(C_{1}\right) \\
\operatorname{vec}\left(C_{2}\right) \\
\operatorname{vec}\left(C_{3}\right)
\end{array}\right], v=\left[\begin{array}{l}
l\left(X_{0}\right) \\
l\left(X_{1}\right) \\
l\left(X_{2}\right) \\
l\left(X_{3}\right)
\end{array}\right] \text {. }
\end{aligned}
$$

Since the equation (11) is equivalent to

$$
\left[\begin{array}{c}
\operatorname{vec}\left(X_{0}\right) \\
\operatorname{vec}\left(X_{1}\right) \\
\operatorname{vec}\left(X_{2}\right) \\
\operatorname{vec}\left(X_{3}\right)
\end{array}\right]=\left[\begin{array}{llll}
E & & & \\
& E & & \\
& & E & \\
& & & E
\end{array}\right]\left[\begin{array}{l}
l\left(X_{0}\right) \\
l\left(X_{1}\right) \\
l\left(X_{2}\right) \\
l\left(X_{3}\right)
\end{array}\right],
$$

then the equation (10) can be represented as

$$
\tilde{G} v=L,
$$

where $v \in \mathrm{R}^{(12 n-8) \times 1}$. Thus, study on the TA matrix solution of problem 1, we have the following results.

Theorem 1. Given $A, C \in \mathrm{Q}^{n \times n}$, then the necessary and sufficient conditions for existence of a TA matrix solution of (1) is $\tilde{G} \tilde{G}^{+} L=L$. When such conditions is satisfied, the general TA matrix solution of (1) is

$$
\begin{gathered}
X=X_{0}+X_{1} i+X_{2} j+X_{3} k, \\
\left\{\begin{array}{l}
v=\tilde{G}^{+} L+\left(I-\tilde{G}^{+} \tilde{G}\right) Y, Y \in \mathrm{R}^{(12 n-8) \times 1}, \\
l\left(X_{0}\right)=v(1: 3 n-2), \\
l\left(X_{1}\right)=v(3 n-1: 6 n-4), \\
l\left(X_{2}\right)=v(6 n-3: 9 n-6), \\
l\left(X_{3}\right)=v(9 n-5: 12 n-8), \\
X_{i}=\operatorname{vec}^{-1}\left(E \cdot l\left(X_{i}\right)\right), \quad i=0,1,2,3,
\end{array}\right.
\end{gathered}
$$

where $F_{1} \in \mathrm{R}^{n^{2} \times(2 n-1)}, F_{2} \in \mathrm{R}^{n^{2} \times(n-1)}$. It is to see that $F_{1}^{*} F_{1}=$ $\operatorname{diag}\left(I_{n}, 2 I_{n-1}\right), F_{2}^{*} F_{2}=2 I_{n-1}$.

Lemma 3. (I) Let $T \in \mathrm{STR}^{n \times n}$ is a real symmetric TA matrix of order $n$, i.e. $b_{i}=c_{i}(i=1, \cdots, n-1)$, then

where $\tilde{G} \in \mathrm{R}^{4 n^{2} \times(12 n-8)}, L \in \mathrm{R}^{4 n^{2} \times 1}$ are represented as (13), $v(1: 3 n-2)$ was regard as $3 n-2$ dimensional vector, $\operatorname{vec}^{-1}(*)$ represents the inverse vec operation.

Proof. From the equation (15) and lemma 2, and we get the necessary and sufficient conditions for existence of a TA matrix solution of (1) is (15) has solutions, which is equivalent to $\tilde{G} \tilde{G}^{+} L=L$. When such conditions is satisfied, the general matrix solution of (15) is

$$
v=\tilde{G}^{+} L+\left(I-\tilde{G}^{+} \tilde{G}\right) Y, \quad \forall Y \in \mathrm{R}^{(12 n-8) \times 1},
$$

form (13), we have

$$
\left\{\begin{array}{l}
l\left(X_{0}\right)=v(1: 3 n-2), \\
l\left(X_{1}\right)=v(3 n-1: 6 n-4), \\
l\left(X_{2}\right)=v(6 n-3: 9 n-6), \\
l\left(X_{3}\right)=v(9 n-5: 12 n-8),
\end{array}\right.
$$

and $\operatorname{vec}\left(X_{i}\right)=E \cdot l\left(X_{i}\right)(i=0,1,2,3)$, where $l(\cdot), E$ are represented as (3), (5). From inverse vec operation, we get $X_{i}=$ $\operatorname{vec}^{-1}\left(E \cdot l\left(X_{i}\right)\right)(i=0,1,2,3$,$) , so the TA matrix solution of$ equation (1) can be given by formula (16). Proof is completed.

Next, we discuss the self-conjugate TA matrix solution of equation (1). Firstly we give a new representation of real symmetric TA matrix and real anti-symmetric TA matrix.

Let

$$
\begin{aligned}
& \left\{\begin{array}{l}
l_{1}(T)=\left(a_{1}, \cdots, a_{n}, b_{1}, \cdots, b_{n-1}\right)^{T} \in \mathrm{R}^{2 n-1}, \\
F_{11}=\operatorname{diag}\left[\begin{array}{llll}
e_{1} & e_{2} & \cdots & e_{n}
\end{array}\right],
\end{array}\right. \\
& \left\{\begin{aligned}
F_{12}= & {\left[\begin{array}{ccccc}
e_{2} & 0 & \cdots & \cdots & 0 \\
e_{1} & \ddots & 0 & & \vdots \\
0 & \ddots & e_{s+1} & \cdots & e_{n} \\
\vdots & \ddots & e_{s} & \ddots & \vdots \\
\vdots & & \ddots & \ddots & 0 \\
0 & \cdots & \cdots & 0 & e_{s}
\end{array}\right], } \\
F_{1} & =\left[\begin{array}{lll}
F_{11} & F_{12}
\end{array}\right],
\end{aligned}\right. \\
& \left\{\begin{array}{l}
l_{2}(T)=\left(b_{1}, \cdots, b_{n-1}\right)^{T} \in \mathrm{R}^{n-1}, \\
F_{2}=\left[\begin{array}{ccccc}
e_{2} & 0 & & \cdots & 0 \\
-e_{1} & \ddots & 0 & \cdots & 0 \\
0 & \ddots & e_{s+1} & \cdots & e_{n} \\
\vdots & \ddots & -e_{s} & & 0 \\
& & & \ddots & \\
0 & \cdots & & 0 & -e_{s}
\end{array}\right],
\end{array}\right.
\end{aligned}
$$




$$
T \in \operatorname{STR}^{n \times n} \Leftrightarrow \operatorname{vec}(T)=F_{1} \cdot l_{1}(T),
$$

where $l_{1}(T), F_{1}$ be as in (20).

(II) Let $T \in \mathrm{ATR}^{n \times n}$ is a real anti-symmetric TA matrix of order $n$, i.e. $b_{i}=-c_{i}(i=1, \cdots, n-1)$ and $a_{i}=0(i=1, \cdots, n)$, then

$$
T \in \operatorname{ATR}^{n \times n} \Leftrightarrow \operatorname{vec}(T)=F_{2} \cdot l_{2}(T),
$$

where $l_{2}(T), F_{2}$ be as in (21).

Let $\breve{X} \in \mathrm{ST}_{n}(\mathrm{Q})$ (self-conjugate TA matrix), its real decomposition is

$$
\breve{X}=X_{0}+X_{1} i+X_{2} j+X_{3} k,
$$

from $\breve{X}=\breve{X}^{*}$, we have $X_{0} \in \mathrm{R}^{n \times n}$ is real symmetric TA matrix $X_{i} \in \mathrm{R}^{n \times n}(i=1,2,3)$ is a real anti-symmetric TA matrix. Thus by lemma 3 , we have

$$
\left\{\begin{array}{l}
\operatorname{vec}\left(X_{0}\right)=F_{1} \cdot l_{1}\left(X_{0}\right), \\
\operatorname{vec}\left(X_{i}\right)=F_{2} \cdot l_{2}\left(X_{i}\right)(i=1,2,3) .
\end{array}\right.
$$

By the quaternion Lyapunov equation (1) is equivalent to equation (10), write

$$
\left\{\begin{array}{c}
\hat{G}=G\left[\begin{array}{lll}
F_{1} & & \\
& F_{2} & \\
& & F_{2} \\
& & F_{2}
\end{array}\right], \\
\hat{L}=\left[\begin{array}{c}
\operatorname{vec}\left(C_{0}\right) \\
\operatorname{vec}\left(C_{1}\right) \\
\operatorname{vec}\left(C_{2}\right) \\
\operatorname{vec}\left(C_{3}\right)
\end{array}\right], \hat{v}=\left[\begin{array}{l}
l_{1}\left(X_{0}\right) \\
l_{2}\left(X_{1}\right) \\
l_{2}\left(X_{2}\right) \\
l_{2}\left(X_{3}\right)
\end{array}\right],
\end{array}\right.
$$

so, the equation (25) is equivalent to

$$
\left[\begin{array}{c}
\operatorname{vec}\left(X_{0}\right) \\
\operatorname{vec}\left(X_{1}\right) \\
\operatorname{vec}\left(X_{2}\right) \\
\operatorname{vec}\left(X_{3}\right)
\end{array}\right]=\left[\begin{array}{llll}
F_{1} & & & \\
& F_{2} & & \\
& & F_{2} & \\
& & & F_{2}
\end{array}\right]\left[\begin{array}{l}
l\left(X_{0}\right) \\
l\left(X_{1}\right) \\
l\left(X_{2}\right) \\
l\left(X_{3}\right)
\end{array}\right],
$$

thus, the equation (10) can be represented as

$$
\hat{G} \hat{v}=\hat{L} .
$$

where $\hat{G} \in \mathrm{R}^{4 n^{2} \times(5 n-4)}, \hat{v} \in \mathrm{R}^{(5 n-4) \times 1}, \hat{L} \in \mathrm{R}^{4 n^{2} \times 1}$. Thus, for the self-conjugate TA matrix solution of problem 1, we have the following results.

Theorem 2. Given $A, C \in \mathrm{Q}^{n \times n}$, then the necessary and sufficient conditions for existence of a self-conjugate TA matrix solution of (1) is $C=C^{*}$ and $\hat{G} \hat{G}^{+} \hat{L}=\hat{L}$, where $\hat{G}, \hat{L}$ be as in (26). When such conditions satisfy, the general solution of (1) is

$$
\hat{X}=X_{0}+X_{1} i+X_{2} j+X_{3} k
$$

where

$$
\left\{\begin{array}{l}
\hat{v}=\hat{G}^{+} \hat{L}+\left(I-\hat{G}^{+} \hat{G}\right) Z, \quad Z \in \mathrm{R}^{(5 n-4) \times 1}, \\
l_{1}\left(X_{0}\right)=\hat{v}(1: 2 n-1), \\
l_{2}\left(X_{1}\right)=\hat{v}(2 n: 3 n-2), \\
l_{2}\left(X_{2}\right)=\hat{v}(3 n-1: 4 n-3), \\
l_{2}\left(X_{3}\right)=\hat{v}(4 n-2: 5 n-4), \\
X_{0}=\operatorname{vec}^{-1}\left(F_{1} \cdot l_{1}\left(X_{0}\right)\right), \\
X_{i}=\operatorname{vec}^{-1}\left(F_{2} \cdot l_{2}\left(X_{i}\right)\right)(i=1,2,3) .
\end{array}\right.
$$

Proof. From the conjugate structure of Lyapunov equation (1), equation (28) and lemma 3, we can get the necessary and sufficient conditions for existence of a self-conjugate TA matrix solution of (1) is (28) has a solution and $C=C^{*}$, which is equivalent to $\hat{G} \hat{G}^{+} \hat{L}=\hat{L}$ and $C=C^{*}$. When such conditions is satisfied, the general solution of the equation (28) is

$$
\hat{v}=\hat{G}^{+} \hat{L}+\left(I-\hat{G}^{+} \hat{G}\right) Z, \quad \forall Z \in \mathrm{R}^{(5 n-4) \times 1},
$$

from (26), we have

$$
\left\{\begin{array}{l}
l_{1}\left(X_{0}\right)=\hat{v}(1: 2 n-1), \\
l_{2}\left(X_{1}\right)=\hat{v}(2 n: 3 n-2), \\
l_{2}\left(X_{2}\right)=\hat{v}(3 n-1: 4 n-3), \\
l_{2}\left(X_{3}\right)=\hat{v}(4 n-2: 5 n-4),
\end{array}\right.
$$

by (25) and inverse vec operation, we get

$$
\left\{\begin{array}{l}
X_{0}=\operatorname{vec}^{-1}\left(F_{1} \cdot l_{1}\left(X_{0}\right)\right), \\
X_{i}=\operatorname{vec}^{-1}\left(F_{2} \cdot l_{2}\left(X_{i}\right)\right)(i=1,2,3),
\end{array}\right.
$$

so the self-conjugate TA matrix solution of equation (1) can be given by the equation (29). Proof is completed.

\section{Solution of Problem 2}

Suppose that the solution set of problem 1 is nonempty, i. e. $\Omega \neq \varnothing, K \in \mathrm{T}_{n}(Q)$, is a known TA matrix. The real decomposition of $K$ is

$$
K=K_{0}+K_{1} i+K_{2} j+K_{3} k,
$$

where $K_{i} \in \mathrm{R}^{n \times n}(i=0,1,2,3)$ are real TA matrices. Write

$$
v_{K}=\left[\begin{array}{c}
l\left(K_{0}\right) \\
l\left(K_{1}\right) \\
l\left(K_{2}\right) \\
l\left(K_{3}\right)
\end{array}\right] \in \mathrm{R}^{(12 n-8) \times 1},
$$

thus, on the solution of problem 2, we have the following results.

Theorem 3. Let $\Omega \neq \varnothing$, given $K \in \mathrm{T}_{n}(Q)$, then there exist 
$\tilde{X} \in \Omega$, such that $\|X-M\|=$ min, and it can be expressed as

$$
\tilde{X}=X_{0}+X_{1} i+X_{2} j+X_{3} k,
$$

where

$$
\left\{\begin{array}{l}
\tilde{v}=\tilde{G}^{+} L+\left(I-\tilde{G}^{+} \tilde{G}\right)\left(I-\tilde{G}^{+} \tilde{G}\right)^{+}\left(v_{K}-\tilde{G}^{+} L\right), \\
l\left(X_{0}\right)=\tilde{v}(1: 3 n-2) \\
l\left(X_{1}\right)=\tilde{v}(3 n-1: 6 n-4), \\
l\left(X_{2}\right)=\tilde{v}(6 n-3: 9 n-6) \\
l\left(X_{3}\right)=\tilde{v}(9 n-5: 12 n-8) \\
X_{i}=\operatorname{vec}^{-1}\left(E \cdot l\left(X_{i}\right)\right), i=0,1,2,3,
\end{array}\right.
$$

the symbolic meaning here is the same as that shown in theorem 1.

Proof. When $X \in \Omega$, based on theorem 1, we notice that the matrix $E$ of (5) is column orthogonal and $E^{*} E=I_{3 n-2}$, so we have

$$
\begin{aligned}
& \|X-K\|^{2}=\sum_{i=0}^{3}\left\|X_{i}-K_{i}\right\|^{2} \\
& =\sum_{i=0}^{3}\left\|\operatorname{vec}\left(X_{i}\right)-\operatorname{vec}\left(K_{i}\right)\right\|^{2} \\
& =\sum_{i=0}^{3}\left\|E \cdot l\left(X_{i}\right)-E \cdot l\left(K_{i}\right)\right\|^{2} \\
& =\sum_{i=0}^{3}\left\|l\left(X_{i}\right)-l\left(K_{i}\right)\right\|^{2} \\
& =\left\|v-v_{K}\right\|^{2} \\
& =\left\|\tilde{G}^{+} L+\left(I-\tilde{G}^{+} \tilde{G}\right) Y-v_{K}\right\|^{2},
\end{aligned}
$$

by (38), we get

$$
\|X-K\|=\min \Leftrightarrow\left\|\left(I-\tilde{G}^{+} \tilde{G}\right) Y-\left(v_{K}-\tilde{G}^{+} L\right)\right\|=\min ,
$$

based on lemma 2, the least squares solution for $Y$ at the right end of (39) is

$$
\tilde{Y}=\left(I-\tilde{G}^{+} \tilde{G}\right)^{+}\left(v_{K}-\tilde{G}^{+} L\right),
$$

so

$$
\begin{aligned}
& \tilde{v}=\tilde{G}^{+} L+\left(I-\tilde{G}^{+} \tilde{G}\right) \tilde{Y} \\
& =\tilde{G}^{+} L+\left(I-\tilde{G}^{+} \tilde{G}\right)\left(I-\tilde{G}^{+} \tilde{G}\right)^{+}\left(v_{K}-\tilde{G}^{+} L\right),
\end{aligned}
$$

for $\tilde{v}$ in (41), write

$$
\left\{\begin{array}{l}
l\left(X_{0}\right)=\tilde{v}(1: 3 n-2), \\
l\left(X_{1}\right)=\tilde{v}(3 n-1: 6 n-4), \\
l\left(X_{2}\right)=\tilde{v}(6 n-3: 9 n-6), \\
l\left(X_{3}\right)=\tilde{v}(9 n-5: 12 n-8),
\end{array}\right.
$$

then we have $\tilde{X}=X_{0}+X_{1} i+X_{2} j+X_{3} k$, where $X_{i}=$ $\operatorname{vec}^{-1}\left(E \cdot l\left(X_{i}\right)\right), \quad i=0,1,2,3$, thus exist $\tilde{X} \in \Omega$ such that $\|X-K\|=\min$ and $\tilde{X}$ is given by (36). Proof is completed.

From the results of theorem 1-3, we give the solution steps for problem 1-2.

(1) Write the real decomposition of the quaternion matrix $A, C$ as (8).

(2) For the selected parameter $s$, a real matrix $\tilde{G}$ and a real vector $L$ are written according to the equation (13), (or a real matrix $\hat{G}$ and a real vector $\hat{L}$ are written according to the equation (26)).

(3) Whether the conditions $\tilde{G} \tilde{G}^{+} L=L$ or $\hat{G} \hat{G}^{+} \hat{L}=\hat{L}$ are met.

i) If the condition is met, it shows that the problem 1 has a solutions. By formula (16), write the solution set $\Omega$ of TA matrix. (or by formula (29), write the solution set $S$ of self-conjugate TA matrix).

ii) If the condition is not met, it shows that the problem 1 has no solution.

(4) When problem 1 has a solution, for given TA matrix $K$, write the corresponding real vector $v_{K}$ by (35).

(5) By (36), write optimal approximation solutions of problem 2. From $\operatorname{vec}\left(X_{i}\right)=T \cdot l\left(X_{i}\right), i=0,1,2,3$, we get four TA real matrices $X_{i}, i=0,1,2,3$, thus $\tilde{X}=X_{0}+X_{1} i+X_{2} j+X_{3} k$.

\section{Numerical Examples}

Example 1. Given quaternion matrices $A, C$ as follows:

$$
A=\left[\begin{array}{cccccc}
0 & 1 & & & & j \\
i & 0 & 1 & & & \\
& i & 0 & 1 & & \\
& & i & 0 & 1 & \\
& & & i & 0 & 1 \\
k & & & & i & 0
\end{array}\right]
$$

$$
C=\left[\begin{array}{cccccc}
-1+i-k & 2 & 1+i & 0 & 0 & 1+j \\
0 & 1+2 i+2 j & 0 & 1+j & -i & -1 \\
2 j & 2 i & 2+j+k & 1+i-k & k & 1 \\
0 & -1+k & 1+j-k & i+k & 1-i+j & 0 \\
0 & j & i-k & i+j+k & 0 & 1+j \\
-1-j & -j & k & j & 1+j & 0
\end{array}\right],
$$

We discuss the TA matrix solution of quaternion Lyapunov equation (1) (here select parameters $s=3$ ).

The real decompositions of quaternion matrices $A$ and $C$ as follows:

$$
A_{0}=\left[\begin{array}{llllll}
0 & 1 & 0 & 0 & 0 & 0 \\
0 & 0 & 1 & 0 & 0 & 0 \\
0 & 0 & 0 & 1 & 0 & 0 \\
0 & 0 & 0 & 0 & 1 & 0 \\
0 & 0 & 0 & 0 & 0 & 1 \\
0 & 0 & 0 & 0 & 0 & 0
\end{array}\right], A_{1}=\left[\begin{array}{llllll}
0 & 0 & 0 & 0 & 0 & 0 \\
1 & 0 & 0 & 0 & 0 & 0 \\
0 & 1 & 0 & 0 & 0 & 0 \\
0 & 0 & 1 & 0 & 0 & 0 \\
0 & 0 & 0 & 1 & 0 & 0 \\
0 & 0 & 0 & 0 & 1 & 0
\end{array}\right],
$$




$$
\begin{aligned}
& A_{2}=\left[\begin{array}{cccccc}
0 & 0 & 0 & 0 & 0 & 1 \\
0 & 0 & 0 & 0 & 0 & 0 \\
0 & 0 & 0 & 0 & 0 & 0 \\
0 & 0 & 0 & 0 & 0 & 0 \\
0 & 0 & 0 & 0 & 0 & 0 \\
0 & 0 & 0 & 0 & 0 & 0
\end{array}\right], A_{3}=\left[\begin{array}{cccccc}
0 & 0 & 0 & 0 & 0 & 0 \\
0 & 0 & 0 & 0 & 0 & 0 \\
0 & 0 & 0 & 0 & 0 & 0 \\
0 & 0 & 0 & 0 & 0 & 0 \\
0 & 0 & 0 & 0 & 0 & 0 \\
1 & 0 & 0 & 0 & 0 & 0
\end{array}\right], \\
& C_{0}=\left[\begin{array}{cccccc}
-1 & 2 & 1 & 0 & 0 & 1 \\
0 & 1 & 0 & 1 & 0 & -1 \\
0 & 0 & 2 & 1 & 0 & 1 \\
0 & -1 & 1 & 0 & 1 & 0 \\
0 & 0 & 0 & 0 & 0 & 1 \\
-1 & 0 & 0 & 0 & 1 & 0
\end{array}\right], C_{1}=\left[\begin{array}{cccccc}
1 & 0 & 1 & 0 & 0 & 0 \\
0 & 2 & 0 & 0 & -1 & 0 \\
0 & 2 & 0 & 1 & 0 & 0 \\
0 & 0 & 0 & 1 & -1 & 0 \\
0 & 0 & 1 & 1 & 0 & 0 \\
0 & 0 & 0 & 0 & 0 & 0
\end{array}\right], \\
& C_{2}=\left[\begin{array}{cccccc}
0 & 0 & 0 & 0 & 0 & 1 \\
0 & 2 & 0 & 1 & 0 & 0 \\
2 & 0 & 1 & 0 & 0 & 0 \\
0 & 0 & 1 & 0 & 1 & 0 \\
0 & 1 & 0 & 1 & 0 & 1 \\
-1 & -1 & 0 & 1 & 1 & 0
\end{array}\right], C_{3}=\left[\begin{array}{cccccc}
-1 & 0 & 0 & 0 & 0 & 0 \\
0 & 0 & 0 & 0 & 0 & 0 \\
0 & 0 & 1 & -1 & 1 & 0 \\
0 & 1 & -1 & 1 & 0 & 0 \\
0 & 0 & -1 & 1 & 0 & 0 \\
0 & 0 & 1 & 0 & 0 & 0
\end{array}\right] .
\end{aligned}
$$

From the equation (15), we write the real matrix $\tilde{G}$ and the real vector $L$. By calculation, we get $\tilde{G} \tilde{G}^{+} L=L$. Thus, by theorem 1, the Lyapunov equation (1) have an unique TA matrix solution $X$, and by formula (16), we obtain

$$
X=\left[\begin{array}{cccccc}
1 & 1+i & 0 & 0 & 0 & 0 \\
j & i & 1 & 0 & 0 & 0 \\
0 & k & 1 & k & 1 & 0 \\
0 & 0 & i & j & 0 & 0 \\
0 & 0 & k & 0 & 1 & 0 \\
0 & 0 & j & 0 & 0 & k
\end{array}\right] .
$$

Example 2. Given quaternion matrices $A, C$ as follows:

$$
\begin{aligned}
A & =\left[\begin{array}{cccccc}
1 & 0 & i & 0 & 0 & k \\
0 & 1 & 0 & i & 0 & 0 \\
j & 0 & 1 & 0 & i & 0 \\
0 & j & 0 & 1 & 0 & i \\
0 & 0 & j & 0 & 1 & 0 \\
k & 0 & 0 & j & 0 & 1
\end{array}\right], \\
C & =\left[\begin{array}{ccccccc}
2 & 1+2 i & 2 i-j & -1-i & k & -1 \\
1-2 i & 2 & 1+i-2 j & i-j & k & j \\
-2 i+j & 1-i+2 j & 2 & 1+k & 3 i-j & -j \\
-1+i & -i+j & 1-k & 2 & -j & i-j \\
-k & -k & -3 i+j & j & 4 & k \\
-1 & -j & j & -i+j & -k & 2
\end{array}\right] .
\end{aligned}
$$

We discuss the self-conjugate TA matrix solution of quaternion Lyapunov equation (1) (here select parameters $s=3)$.
The real decomposition of quaternion matrices $A$ and $C$ as follows:

$$
\begin{aligned}
& A_{0}=\left[\begin{array}{llllll}
1 & 0 & 0 & 0 & 0 & 0 \\
0 & 1 & 0 & 0 & 0 & 0 \\
0 & 0 & 1 & 0 & 0 & 0 \\
0 & 0 & 0 & 1 & 0 & 0 \\
0 & 0 & 0 & 0 & 1 & 0 \\
0 & 0 & 0 & 0 & 0 & 1
\end{array}\right], A_{1}=\left[\begin{array}{llllll}
0 & 0 & 1 & 0 & 0 & 0 \\
0 & 0 & 0 & 1 & 0 & 0 \\
0 & 0 & 0 & 0 & 1 & 0 \\
0 & 0 & 0 & 0 & 0 & 1 \\
0 & 0 & 0 & 0 & 0 & 0 \\
0 & 0 & 0 & 0 & 0 & 0
\end{array}\right], \\
& A_{2}=\left[\begin{array}{llllll}
0 & 0 & 0 & 0 & 0 & 0 \\
0 & 0 & 0 & 0 & 0 & 0 \\
1 & 0 & 0 & 0 & 0 & 0 \\
0 & 1 & 0 & 0 & 0 & 0 \\
0 & 0 & 1 & 0 & 0 & 0 \\
0 & 0 & 0 & 1 & 0 & 0
\end{array}\right], A_{3}=\left[\begin{array}{llllll}
0 & 0 & 0 & 0 & 0 & 1 \\
0 & 0 & 0 & 0 & 0 & 0 \\
0 & 0 & 0 & 0 & 0 & 0 \\
0 & 0 & 0 & 0 & 0 & 0 \\
0 & 0 & 0 & 0 & 0 & 0 \\
1 & 0 & 0 & 0 & 0 & 0
\end{array}\right], \\
& C_{0}=\left[\begin{array}{cccccc}
2 & 1 & 0 & -1 & 0 & -1 \\
1 & 2 & 1 & 0 & 0 & 0 \\
0 & 1 & 2 & 1 & 0 & 0 \\
-1 & 0 & 1 & 2 & 0 & 0 \\
0 & 0 & 0 & 0 & 4 & 0 \\
-1 & 0 & 0 & 0 & 0 & 2
\end{array}\right], C_{1}=\left[\begin{array}{cccccc}
0 & 2 & 2 & -1 & 0 & 0 \\
-2 & 0 & 1 & 1 & 0 & 0 \\
-2 & -1 & 0 & 0 & 3 & 0 \\
1 & -1 & 0 & 0 & 0 & 1 \\
0 & 0 & -3 & 0 & 0 & 0 \\
0 & 0 & 0 & -1 & 0 & 0
\end{array}\right], \\
& C_{2}=\left[\begin{array}{cccccc}
0 & 0 & -1 & 0 & 0 & 0 \\
0 & 0 & -2 & -1 & 0 & 1 \\
1 & 2 & 0 & 0 & -1 & -1 \\
0 & 1 & 0 & 0 & -1 & -1 \\
0 & 0 & 1 & 1 & 0 & 0 \\
0 & -1 & 1 & 1 & 0 & 0
\end{array}\right], C_{3}=\left[\begin{array}{cccccc}
0 & 0 & 0 & 0 & 1 & 0 \\
0 & 0 & 0 & 0 & 1 & 0 \\
0 & 0 & 0 & 1 & 0 & 0 \\
0 & 0 & -1 & 0 & 0 & 0 \\
-1 & -1 & 0 & 0 & 0 & 1 \\
0 & 0 & 0 & 0 & -1 & 0
\end{array}\right] \text {. }
\end{aligned}
$$

From the equation (28), we write the real matrix $\hat{G}$ and the real vector $\hat{L}$ and $C=C^{*}$. By calculation, we get $\hat{G} \hat{G}^{+} \hat{L}=\hat{L}$. Thus, by theorem 2, the Lyapunov equation (1) have an unique self-conjugate TA matrix solution $\hat{X}$, and by formula (29), we obtain

$$
\hat{X}=\left[\begin{array}{cccccc}
1 & i & 0 & 0 & 0 & 0 \\
-i & 1 & -j & 0 & 0 & 0 \\
0 & j & 1 & k & i & -j \\
0 & 0 & -k & 1 & 0 & 0 \\
0 & 0 & -i & 0 & 1 & 0 \\
0 & 0 & j & 0 & 0 & 1
\end{array}\right] .
$$

\section{Conclusion}

In this paper, the solution to quaternion Lyapunov equation with tridiagonal-arrow matrix constraints is discussed. Firstly, a new characterization of TA matrix and self-conjugate TA matrix is given by using matrix straighten operator. Then, the constrained equation is transformed into a unconstrained matrix equation by using the real decomposition of quaternion 
matrix and the Kronecker product of real matrix. Thus, the necessary and sufficient conditions for the solution to problem 1 and its general solution expression are obtained. Finally, by using invariance of Frobenius norm of orthogonal matrix product, the optimal approximation solution of problem 2 is obtained under the condition of problem 1 solution set $\Omega \neq \varnothing$. This paper offers an effective method to solve the problem of related constrained quaternion matrix.

\section{Acknowledgements}

This work is supported by the national natural science foundation of china (Grant No. 11661011); Innovation project of Guangxi university for nationalities graduate education (Grant No. gxun-chxzs2019030).

\section{References}

[1] Z. Gajic, and M. T. J. Qureshi, Lyapunov Matrix Equation in System Stability and Control, Academic Press, 1995.

[2] Y. M. Fu, and G. R. Duan, "Robust stability analysis for uncertain systems with time-varying delays," J Nat Sci Heilongjiang Univ, vol. 22, pp. 655-658, May 2005.

[3] Z. F. Feng, "Study on network of temperature sensors via Lyapunov optimization," Master Thesis, South China University of Technology, 2017.

[4] Q. Hui, and J. M. Berg, "Semistability theory for spatially distributed systems," Syst Control Lett, 2013, vol. 62, pp. 862-870.

[5] A. L. Afflitto, W. M. Haddad, and Q. Hui, "Optimal control for linear and nonlinear semistabilization," J Franklin I, vol. 352, pp. 851-881, March 2015.
[6] V. Simoncini, "A new iterative method for solving large-scale Lyapunov matrix equations,” SIAM J Sci Comp, 2007, vol. 29, pp. $1268-1288$.

[7] S. F. Xu, and M. S. Cheng, "On the solvability for the mixed-type Lyapunov equation," IMA J Appl Math, vol. 71, pp. 287-294.

[8] Y. Deng, and J. P. Huang, "The subpositive definite solution of the mixed-type Lyapunov equation over quaternion field," Acta Sci Natur Univ Nankai, 2011, vol. 44, pp. 41-46.

[9] J. P. Huang, Y. S. Lu, and K. J. Xu, "On cyclic matrix solution of unified algebraic Lyapunov equation and its optimal approximation over quaternion field," J. Southwest China Normal Univ (Nat Sci Ed), vol. 41, pp. 1-5, April 2016.

[10] G. H. Golub, and C. F. Van Loan, Matrix Computations, The Johns Hopkins University Press, 1996.

[11] K. Atkinson, and W. M. Han, Theoretical Numerical Analysis, New York: Springer-Verlag, 2009.

[12] Y. Yu, J. H. Yuan, and J. Qian, "Cubic spline interpolation with new boundary conditions," Comput Eng Softw, 2016, vol. 37, pp. 25-28.

[13] H. B. Li, and E. J. Zhong, "A note on semi-discrete Difference schemes of heat conduction equation," Math Num Sin, vol. 37, pp. 401-414, April 2015.

[14] L. L. Ma, and J. P. Huang, "On the construction of arrow-like quaternion matrices from two right eigenpairs," ICMTA2010, Shanghai, China, July, 2010, pp. 280-284.

[15] J. P. Huang, J. X. Lan, and L. Y. Mao, "The solutions of quaternion sylvester equation with arrowhead matrix constraint," J Math Practice Theory, 2018, vol. 48, pp. 264-271.

[16] F. J. Duan, T. Fang, and F. Yuan, "Inverse eigenvalue problems for a class of special matrices," J Math, 2018. DOI: 10.13548/j.sxzz.20181206.001. 